PROCEEDINGS OF THE

AMERICAN MATHEMATICAL SOCIETY

Volume 130, Number 10, Pages 2985-2994

S 0002-9939(02)06410-9

Article electronically published on March 14, 2002

\title{
CONTINUITY AND DIFFERENTIABILITY FOR WEIGHTED SOBOLEV SPACES
}

\author{
YOSHIHIRO MIZUTA AND TETSU SHIMOMURA
}

(Communicated by Juha M. Heinonen)

\begin{abstract}
Our aim in this paper is to discuss continuity and differentiability of functions in weighted Sobolev spaces in the limiting case of Sobolev's imbedding theorem.
\end{abstract}

\section{Introduction AND STATEMENT OF RESUlts}

Let $X$ be a metric space with a metric $d$. We denote by $B(x, r)$ the open ball centered at $x \in X$ with radius $r>0$. For $\sigma>0$, we write

$$
\sigma B(x, r)=B(x, \sigma r) \text {. }
$$

Let $\mu$ be a Borel measure on $X$. Assume that there exist constants $C>0$ and $s \geqq 1$ such that

$$
\frac{\mu\left(B^{\prime}\right)}{\mu(B)} \geqq C\left(\frac{r^{\prime}}{r}\right)^{s}
$$

for all balls $B=B(x, r)$ and $B^{\prime}=B\left(x^{\prime}, r^{\prime}\right)$ with $x^{\prime} \in B$ and $0<r^{\prime} \leqq r$. Note that $\mu$ is a doubling measure on $X$, that is, there exists a constant $C^{\prime}>0$ such that

$$
\mu(B(x, 2 r)) \leqq C^{\prime} \mu(B(x, r))
$$

for all $x \in X$ and $r>0$.

We say that a pair $(u, g)$ of functions in $L_{l o c}^{p}(X ; \mu)$ satisfies a $p$-Poincaré inequality (on rings), $1 \leqq p<\infty$, if for every $c_{1}$ and $c_{2}$ with $c_{2}>c_{1}>1$ there are constants $M>0$ and $\sigma \geqq 1$ such that

$$
f_{A\left(r, r^{\prime}\right)}\left|u(y)-u_{A\left(r, r^{\prime}\right)}\right| d \mu \leqq M r\left(f_{\sigma A\left(r, r^{\prime}\right)}|g|^{p} d \mu\right)^{1 / p}
$$

whenever $c_{1} r^{\prime}<r<c_{2} r^{\prime}$, where $A\left(r, r^{\prime}\right)=B(x, r)-B\left(x, r^{\prime}\right), \sigma A\left(r, r^{\prime}\right)=B(x, \sigma r)-$ $B\left(x, \sigma^{-1} r^{\prime}\right)$ and

$$
u_{G}=f_{G} u d \mu=\frac{1}{\mu(G)} \int_{G} u d \mu
$$

for Borel sets $G \subset X$. If (1.3) holds for $r^{\prime}=0$, then the pair $(u, g)$ is said to satisfy the usual $p$-Poincaré inequality on balls. Under certain assumptions, the

Received by the editors December 18, 2000 and, in revised form, May 9, 2001.

2000 Mathematics Subject Classification. Primary 46E35.

Key words and phrases. Hölder continuity, differentiability, weighted Sobolev spaces, $A_{p^{-}}$ weight, $p$-Poincaré inequality, Sobolev's imbedding theorem. 
usual $p$-Poincaré inequality on balls implies our $p$-Poincaré inequality; see e.g. [2] Theorem 9.7].

A positive measurable function $w$ on $\mathbf{R}^{n}$ is called an $A_{p}$ weight (written as $\left.w \in\left(A_{p}\right)\right)$ if there exists a positive constant $C_{p}$ such that

$$
f_{B} w(x) d x\left(f_{B} w(x)^{1 /(1-p)} d x\right)^{p-1} \leqq C_{p} \quad(<\infty)
$$

for all balls $B$, where $1<p<\infty$ and

$$
f_{B} w(x) d x=\frac{1}{\mathcal{L}^{n}(B)} \int_{B} w(x) d x
$$

with $\mathcal{L}^{n}$ denoting the $n$-dimensional Lebesgue measure; we say that $w$ is an $A_{1}$ weight if there exists a positive constant $C_{1}$ such that

$$
f_{B} w(x) d x \leqq C_{1} \operatorname{essinf}_{B} w
$$

for all balls $B$. Note that if $w$ is an $A_{p}$ weight, then $d \mu=w d x$ satisfies the decay condition (1.1) with $s=n p$, due to [3] Section 15.5].

In view of [3, Section 15.26], we can prove that the $p$-Poincaré inequality is valid for $(u,|\nabla u|)$ and $d \mu=w d x$ with $u \in W^{1, p}\left(\mathbf{R}^{n} ; \mu\right)$ and $w \in\left(A_{p}\right)$, where $W^{1, p}\left(\mathbf{R}^{n} ; \mu\right)$ denotes the weighted Sobolev space, which is

$$
W^{1, p}\left(\mathbf{R}^{n} ; \mu\right)=\left\{u \in L^{p}\left(\mathbf{R}^{n} ; \mu\right):|\nabla u| \in L^{p}\left(\mathbf{R}^{n} ; \mu\right)\right\}
$$

for this fact, see Section 3 below.

Recently Björn ([1, Theorem 1.5 and Theorem 3.1]) proved the following theorem:

Theorem A. Let $\mu$ be a Borel measure on $\mathbf{R}^{n}$ satisfying (1.1). Let $u$ be a function in the Sobolev space $W^{1, p}\left(\mathbf{R}^{n} ; \mu\right)$ such that $(u,|\nabla u|)$ satisfies the usual p-Poincaré inequality on balls. If $p>s$, then $u$ can be modified on a set of $\mu$-measure zero so that it is locally Hölder continuous in $\mathbf{R}^{n}$ and totally differentiable $\mu$-a.e. in $\mathbf{R}^{n}$.

In case $\mu$ is the Lebesgue measure in $\mathbf{R}^{n}$, the proof of Theorem $\mathrm{A}$ is given in Stein [9, Theorem 1, Chap. VIII]. Our aim in this paper is to discuss the Hölder continuity and differentiability of Sobolev functions in the limiting case $p=s$. To do so, consider a positive function $\Phi_{p}(r)$ on $\mathbf{R}_{+}=[0, \infty)$ with the following properties:

$(\varphi 1) \Phi_{p}(r)$ is of the form $r^{p} \varphi(r)$, where $1<p<\infty$ and $\varphi$ is a positive nondecreasing function on $(0, \infty)$. The value $\Phi_{p}(0)$ is defined to be zero.

$(\varphi 2)$ There exists $c>1$ such that

$$
c^{-1} \varphi(r) \leqq \varphi\left(r^{2}\right) \leqq c \varphi(r) \quad \text { whenever } r>0
$$

$(\varphi 3) \varphi^{*}(1)<\infty$, where

$$
\varphi^{*}(r)=\left(\int_{0}^{r}\left[\varphi\left(t^{-1}\right)\right]^{-p^{\prime} / p} t^{-1} d t\right)^{1 / p^{\prime}}, \quad \frac{1}{p}+\frac{1}{p^{\prime}}=1 .
$$

Typical examples of $\varphi$ are

$$
[\log (1+r)]^{\delta}, \quad[\log (1+r)]^{p-1}[\log (1+\log (1+r))]^{\delta}, \quad \cdots
$$

for $\delta>p-1$.

Our first aim is to prove the following result (cf. [1] Theorem 3.1], [2, Theorem $5.1])$. 
Theorem 1. Let $X$ be a connected metric space and let $\mu$ be a Borel measure on $X$ satisfying the decay condition (1.1) with $s=p, 1<p<\infty$. Assume that a pair $(u, g)$ satisfies the $p$-Poincaré inequality in $X$ and

$$
\int_{X} \Phi_{p}(|g(x)|) d \mu(x)<\infty
$$

Then $u$ can be modified on a set of $\mu$-measure zero so that it is locally $\varphi^{*}$-Hölder continuous on $X$. Moreover, $u$ satisfies

$$
|u(x)-u(y)| \leqq M r_{0} \varphi^{*}(r)\left(\int_{c \sigma B_{0}} \Phi_{p}(|g|) d \mu\right)^{1 / p}+M r^{1-\varepsilon}
$$

for all $x, y \in B=B\left(x_{0}, r\right)$ and $B_{0}=B\left(x_{0}, r_{0}\right)$ with $0<r<r_{0}$, where $c=2^{4}+1=$ $17,0<\varepsilon<1$ and $M$ is a positive constant depending on $\varepsilon$.

Corollary 1. Let $1<p<\infty$ and $w \in\left(A_{p}\right)$. Assume further that $\mu$ satisfies (1.1) with $s=p$, where $d \mu=w d x$. Let $u$ be a function in $W^{1, p}\left(\mathbf{R}^{n} ; \mu\right)$ satisfying

$$
\int_{\mathbf{R}^{n}} \Phi_{p}(|\nabla u(x)|) d \mu(x)<\infty .
$$

Then $u$ can be modified on a set of measure zero so that it becomes a locally $\varphi^{*}$ Hölder continuous function on $\mathbf{R}^{n}$ satisfying

$$
|u(x)-u(y)| \leqq M r_{0} \varphi^{*}(r)\left(f_{c B_{0}} \Phi_{p}(|\nabla u|) d \mu\right)^{1 / p}+M r^{1-\varepsilon}
$$

for all $x, y \in B=B\left(x_{0}, r\right)$ and $B_{0}=B\left(x_{0}, r_{0}\right)$ with $0<r<r_{0}$, where $c=2^{4}+1=$ $17,0<\varepsilon<1$ and $M$ is a positive constant depending on $\varepsilon$.

In view of [4] Theorem 1], we know that the Riesz potentials $U_{\alpha} f$ are locally $\varphi^{*}$-Hölder continuous on $R^{n}$ when $U_{\alpha}|f| \not \equiv \infty$ and $\int_{\mathbf{R}^{n}} \Phi_{p}(|f(y)|) d y<\infty$ with $p=n / \alpha>1$. In particular, if $u \in W^{1, n}\left(\mathbf{R}^{n}\right)$ satisfies 1.5 with $\mu=\mathcal{L}^{n}$ and $p=n$, then $u$ can be modified on a set of measure zero so that it becomes locally $\varphi^{*}$-Hölder continuous on $\mathbf{R}^{n}$. By [7, Remark 3.3], we know that if $\varphi^{*}(1)=\infty$, then we can find an $f$ satisfying $U_{\alpha}|f| \not \equiv \infty$ and $\int_{\mathbf{R}^{n}} \Phi_{p}(|f(y)|) d y<\infty$ with $p=n / \alpha>1$ such that $U_{\alpha}|f|(0)=\infty$, so that $U_{\alpha} f$ is not continuous at the origin in the usual sense.

We say that a function $u$ on $\mathbf{R}^{n}$ is totally differentiable at $x_{0}$ if

$$
\lim _{x \rightarrow x_{0}} \frac{\left|u(x)-u\left(x_{0}\right)-a \cdot\left(x-x_{0}\right)\right|}{\left|x-x_{0}\right|}=0
$$

for some $a \in \mathbf{R}^{n}$.

Theorem 2. Let $1<p<\infty$ and $w \in\left(A_{p}\right)$. Assume further that $\mu$ satisfies (1.1) with $s=p$, where $d \mu=w d x$. Let $u$ be a function in $W^{1, p}\left(\mathbf{R}^{n} ; \mu\right)$ satisfying (1.5). Then $u$ can be modified on a set of measure zero so that it becomes totally differentiable a.e. on $\mathbf{R}^{n}$.

Theorem 2 was proved by the first author [6, Theorem 3.2] in the case $\mu=\mathcal{L}^{n}$ and $p=n$ (see also [4, Theorem 2] and [7] Theorem 3.2]). 


\section{LEMMAS}

Throughout this paper, let $M$ denote various positive constants independent of the variables in question and $M(\varepsilon)$ denote a positive constant which depends on $\varepsilon$.

Let $u \in L_{l o c}^{1}(X ; \mu)$. We say that $x \in X$ is a Lebesgue point of $u$ if

$$
\lim _{r \rightarrow 0} f_{B(x, r)}|u(y)-u(x)| d \mu(y)=0 .
$$

Lemma 1. Let $\mu$ be a Borel measure on a connected metric space $X$ with the decay condition (1.1), and $u \in L_{l o c}^{1}(X ; \mu)$. If $x \in X$ is a Lebesgue point of $u$ and $0<c<1$, then

$$
\lim _{r \rightarrow 0} f_{A(r)}|u(y)-u(x)| d \mu(y)=0
$$

where $A(r)=B(r)-B(c r)$ with $B(r)=B(x, r)$.

Proof. Since $X$ is connected, we can find $y$ such that $d(x, y)=(r+c r) / 2=$ $(1+c) r / 2$. Let $B^{\prime}=B(y,(1-c) r / 2)$. By the lower bound (1.1), we have

$$
\frac{\mu\left(B^{\prime}\right)}{\mu(B(r))} \geqq C\left(\frac{(1-c) r / 2}{r}\right)^{s}=C((1-c) / 2)^{s} .
$$

Since $B^{\prime} \subset A(r) \subset B(r)$, we have

$$
f_{A(r)}|u(y)-u(x)| d \mu(y) \leqq M f_{B(r)}|u(y)-u(x)| d \mu(y) \rightarrow 0
$$

as $r \rightarrow 0$, by the assumption that $x$ is a Lebesgue point of $u$. Thus the present lemma is obtained.

Lemma 2. Let $\mu$ be a Borel measure on $X$. Let $A$ and $B$ be Borel sets such that $A \subset B$ and $0<\mu(B) \leqq M \mu(A)$. Then

$$
\left|u_{A}-u_{B}\right| \leqq M f_{B}\left|u-u_{B}\right| d \mu .
$$

Proof. Since $A \subset B$ and $\mu(B) \leqq M \mu(A)$, we have

$$
\left|u_{A}-u_{B}\right| \leqq f_{A}\left|u-u_{B}\right| d \mu \leqq M f_{B}\left|u-u_{B}\right| d \mu
$$

as required.

Corollary 2. Let $\mu$ be a Borel measure on $X$. Let $A, B$ and $C$ be Borel sets such that $A \subset C, B \subset C, 0<\mu(C) \leqq M \mu(A)$ and $0<\mu(C) \leqq M \mu(B)$. Then

$$
\left|u_{A}-u_{B}\right| \leqq 2 M f_{C}\left|u-u_{C}\right| d \mu .
$$

In fact, by Lemma 2 , we see that

$$
\begin{aligned}
\left|u_{A}-u_{B}\right| & \leqq\left|u_{A}-u_{C}\right|+\left|u_{B}-u_{C}\right| \\
& \leqq 2 M f_{C}\left|u-u_{C}\right| d \mu .
\end{aligned}
$$

For every ball $B=B\left(x_{0}, r\right), x \in B$, and $\sigma \geqq 1$, put $A_{i}=A_{i}(x)=B\left(x, 2^{i}\right)-$ $B\left(x, 2^{i-j_{0}}\right)$, where $j_{0}$ is the integer such that $2^{j_{0}+1}>4 \sigma^{2} \geqq 2^{j_{0}}$. Let $i_{0}$ be the integer such that $\sigma 2^{i_{0}+1}>2^{j_{0}+2} r \geqq \sigma 2^{i_{0}}$. 
Lemma 3. Let $\mu$ be a doubling measure on a connected metric space $X$. If a pair $(u, g)$ satisfies the $p$-Poincaré inequality in $X$, then

$$
\left|u(x)-u_{\sigma^{-1} A_{i_{0}}}\right| \leqq M \sum_{i=-\infty}^{i_{0}} 2^{i}\left(f_{\tilde{A}_{i}}|g|^{p} d \mu\right)^{1 / p}
$$

holds for almost every $x \in B$, where $\tilde{A}_{i}=A_{i} \cup A_{i-1}=B\left(x, 2^{i}\right)-B\left(x, 2^{i-j_{0}-1}\right)$.

Proof. Let $x \in B$ be a Lebesgue point of $u$. Note that $u_{\sigma^{-1}} A_{i} \rightarrow u(x)$ as $i \rightarrow-\infty$ by Lemma 1 . Using Lemma 2 and the doubling property of $\mu$, we obtain

$$
\left|u(x)-u_{\sigma^{-1} A_{i_{0}}}\right| \leqq \sum_{i=-\infty}^{i_{0}}\left|u_{\sigma^{-1} A_{i}}-u_{\sigma^{-1} A_{i-1}}\right| \leqq M \sum_{i=-\infty}^{i_{0}} f_{\sigma^{-1} \tilde{A}_{i}}\left|u-u_{\sigma^{-1} \tilde{A}_{i}}\right| d \mu .
$$

By the $p$-Poincaré inequality, we obtain

$$
\left|u(x)-u_{\sigma^{-1} A_{i_{0}}}\right| \leqq M \sum_{i=-\infty}^{i_{0}} \sigma^{-1} 2^{i}\left(f_{\tilde{A}_{i}}|g|^{p} d \mu\right)^{1 / p},
$$

as required.

Lemma 4. Let $\mu$ and $(u, g)$ be as above. Then

$$
\left|u_{\sigma^{-1} A_{i_{0}}}-u_{\tilde{A}}\right| \leqq M 2^{i_{0}}\left(f_{\sigma \tilde{A}}|g|^{p} d \mu\right)^{1 / p}
$$

holds for almost every $x \in B$ and $\tilde{A}=B\left(x_{0}, c r\right)-B\left(x_{0}, r\right)$ with $c=2^{4}+1$.

In fact, since $\sigma^{-1} A_{i_{0}} \subset \tilde{A}$, Lemma 4 follows readily from Lemma 2 and the $p$-Poincaré inequality.

Lemma 5. Let $g$ be a measurable function on $X$ satisfying

$$
\int_{X} \Phi_{p}(g(y)) d \mu(y)<\infty
$$

Then

$$
\lim _{r \rightarrow 0} f_{B(x, r)} \Phi_{p}(|g(y)-g(x)|) d \mu(y)=0
$$

for $\mu$-a.e. $x \in X$.

To prove this, it suffices to note that

$$
\Phi_{p}(a) \leqq \Phi_{p}(a+b)-\Phi_{p}(b)
$$

for all $a \geqq 0$ and $b \geqq 0$.

\section{Proofs of Theorems 1 And 2}

First we collect properties which follow from conditions $(\varphi 1)$ and $(\varphi 2)$ (see [5. Section 2] and [8 Section 2]).

$(\varphi 4)$ The function $\varphi$ satisfies the doubling condition, that is, there exists $c>1$ such that

$$
c^{-1} \varphi(r) \leqq \varphi(2 r) \leqq c \varphi(r) \quad \text { whenever } r>0 .
$$

$(\varphi 5)$ For any $\gamma>0$, there exists $c=c(\gamma) \geqq 1$ such that

$$
c^{-1} \varphi(r) \leqq \varphi\left(r^{\gamma}\right) \leqq c \varphi(r) \quad \text { whenever } r>0
$$


( $\varphi 6)$ If $\gamma>0$, then there exists $c=c(\gamma) \geqq 1$ such that

$$
s^{\gamma} \varphi\left(s^{-1}\right) \leqq c t^{\gamma} \varphi\left(t^{-1}\right) \quad \text { whenever } 0<s<t .
$$

Proof of Theorem 1. By Lemma 3, we have for every ball $B$ with radius $r, 0<\varepsilon<1$ and $\delta>0$

$$
\begin{aligned}
\mid u(x)-u_{\sigma^{-1} A_{i_{0}} \mid \leqq} & M \sum_{i=-\infty}^{i_{0}} 2^{i} \mu\left(\tilde{A}_{i}\right)^{-1 / p}\left(\int_{\tilde{A}_{i}}|g(y)|^{p} d \mu(y)\right)^{1 / p} \\
= & M \sum_{i=-\infty}^{i_{0}} 2^{i} \mu\left(\tilde{A}_{i}\right)^{-1 / p}\left(\int_{\left\{y \in \tilde{A}_{i}:|g(y)|>\left(\delta 2^{-i}\right)^{\varepsilon}\right\}}|g(y)|^{p} d \mu\right)^{1 / p} \\
& +M \sum_{i=-\infty}^{i_{0}} 2^{i} \mu\left(\tilde{A}_{i}\right)^{-1 / p}\left(\int_{\left\{y \in \tilde{A}_{i}:|g(y)| \leqq\left(\delta 2^{-i}\right)^{\varepsilon}\right\}}|g(y)|^{p} d \mu\right)^{1 / p} \\
= & M u_{1}+M u_{2} .
\end{aligned}
$$

By condition $(\varphi 5)$, we see that if $|g(y)|>\left(\delta 2^{-i}\right)^{\varepsilon}$, then

$$
\varphi(|g|) \geqq \varphi\left(\left(\delta 2^{-i}\right)^{\varepsilon}\right) \geqq M(\varepsilon) \varphi\left(\delta 2^{-i}\right) .
$$

By the lower bound (1.1) with $s=p$, we have

$$
\mu\left(\tilde{A}_{i}\right) \geqq M \mu(B)\left(\frac{2^{i}}{r}\right)^{p}
$$

so that

$$
\begin{aligned}
u_{1} & \leqq M \sum_{i=-\infty}^{i_{0}} 2^{i} \mu\left(\tilde{A}_{i}\right)^{-1 / p}\left[\varphi\left(\delta 2^{-i}\right)\right]^{-1 / p}\left(\int_{\tilde{A}_{i}} \Phi_{p}(|g|) d \mu\right)^{1 / p} \\
& \leqq M \sum_{i=-\infty}^{i_{0}} 2^{i} \mu(B)^{-1 / p}\left(\frac{2^{i}}{r}\right)^{-1}\left[\varphi\left(\delta 2^{-i}\right)\right]^{-1 / p}\left(\int_{\tilde{A}_{i}} \Phi_{p}(|g|) d \mu\right)^{1 / p} \\
& \leqq M r \mu(B)^{-1 / p} \sum_{i=-\infty}^{i_{0}}\left[\varphi\left(\delta 2^{-i}\right)\right]^{-1 / p}\left(\int_{\tilde{A}_{i}} \Phi_{p}(|g|) d \mu\right)^{1 / p}
\end{aligned}
$$

On the other hand, we have

$$
\begin{aligned}
u_{2} & \leqq M \sum_{i=-\infty}^{i_{0}} 2^{i} \mu\left(\tilde{A}_{i}\right)^{-1 / p}\left(\delta 2^{-i}\right)^{\varepsilon} \mu\left(\tilde{A}_{i}\right)^{1 / p} \\
& =M \sum_{i=-\infty}^{i_{0}}\left(2^{i}\right)^{1-\varepsilon} \delta^{\varepsilon} \\
& \leqq M r^{1-\varepsilon} \delta^{\varepsilon}
\end{aligned}
$$

Hence we establish

$$
\begin{aligned}
\left|u(x)-u_{\sigma^{-1} A_{i_{0}}}\right| \leqq & M r \mu(B)^{-1 / p} \sum_{i=-\infty}^{i_{0}}\left[\varphi\left(\delta 2^{-i}\right)\right]^{-1 / p}\left(\int_{\tilde{A}_{i}} \Phi_{p}(|g|) d \mu\right)^{1 / p} \\
+ & M r^{1-\varepsilon} \delta^{\varepsilon}
\end{aligned}
$$

where $M=M(\varepsilon)$ is a positive constant independent of $\delta, r$ and $x$. 
From Hölder's inequality, we obtain

$$
\begin{aligned}
& \mid u(x)-u_{\sigma^{-1} A_{i_{0}} \mid} \\
& \quad \leqq M r \mu(B)^{-1 / p}\left(\sum_{i=-\infty}^{i_{0}}\left[\varphi\left(\delta 2^{-i}\right)\right]^{-p^{\prime} / p}\right)^{1 / p^{\prime}}\left(\sum_{i=-\infty}^{i_{0}} \int_{\tilde{A}_{i}} \Phi_{p}(|g|) d \mu\right)^{1 / p}+M r^{1-\varepsilon} \delta^{\varepsilon},
\end{aligned}
$$

where $1 / p+1 / p^{\prime}=1$. By condition $(\varphi 4)$, we see that

$$
\left(\sum_{i=-\infty}^{i_{0}}\left[\varphi\left(\delta 2^{-i}\right)\right]^{-p^{\prime} / p}\right)^{1 / p^{\prime}} \leqq M \varphi^{*}\left(2^{4} \sigma r \delta^{-1}\right) \leqq M \varphi^{*}\left(r \delta^{-1}\right) .
$$

Since $\tilde{A}_{i} \subset c \sigma B$ for $i \leqq i_{0}$ and $c=2^{4}+1$, it follows that

$$
\begin{aligned}
& \mid u(x)-u_{\sigma^{-1} A_{i_{0}} \mid} \leqq M r \mu(B)^{-1 / p} \varphi^{*}\left(r \delta^{-1}\right)\left(\int_{\cup A_{i}} \Phi_{p}(|g|) d \mu\right)^{1 / p}+M r^{1-\varepsilon} \delta^{\varepsilon} \\
& \leqq M r \mu(B)^{-1 / p} \varphi^{*}\left(r \delta^{-1}\right)\left(\int_{c \sigma B} \Phi_{p}(|g|) d \mu\right)^{1 / p}+M r^{1-\varepsilon} \delta^{\varepsilon} \\
& \leqq M r \varphi^{*}\left(r \delta^{-1}\right)\left(f_{c \sigma B} \Phi_{p}(|g|) d \mu\right)^{1 / p}+M r^{1-\varepsilon} \delta^{\varepsilon} .
\end{aligned}
$$

Next we estimate $\left|u_{\sigma^{-1} A_{i_{0}}}-u_{\tilde{A}}\right|$ by Lemma 4 . Noting that by condition $(\varphi 4)$

$$
\varphi^{*}\left(\delta^{-1} r\right) \geqq\left(\int_{\frac{1}{2} \delta^{-1} r}^{\delta^{-1} r}\left[\varphi\left(t^{-1}\right)\right]^{-p^{\prime} / p} t^{-1} d t\right)^{1 / p^{\prime}} \geqq M\left[\varphi\left(\delta r^{-1}\right)\right]^{-1 / p},
$$

we have

$$
\begin{aligned}
\left|u_{\sigma^{-1} A_{i_{0}}}-u_{\tilde{A}}\right| & \leqq M 2^{i_{0}}\left(f_{\sigma \tilde{A}}|g|^{p} d \mu\right)^{1 / p} \\
& \leqq M r\left[\varphi\left(\delta r^{-1}\right)\right]^{-1 / p}\left(f_{c \sigma B} \Phi_{p}(|g|) d \mu\right)^{1 / p}+M r^{1-\varepsilon} \delta^{\varepsilon} \\
& \leqq M r \varphi^{*}\left(\delta^{-1} r\right)\left(f_{c \sigma B} \Phi_{p}(|g|) d \mu\right)^{1 / p}+M r^{1-\varepsilon} \delta^{\varepsilon} .
\end{aligned}
$$

By the lower bound (1.1), we have

$$
\begin{aligned}
|u(x)-u(y)| \leqq & \left|u(x)-u_{\sigma^{-1} A_{i_{0}}(x)}\right|+\left|u_{\sigma^{-1} A_{i_{0}}(x)}-u_{\tilde{A}}\right| \\
& +\left|u_{\tilde{A}}-u_{\sigma^{-1} A_{i_{0}}(y)}\right|+\left|u_{\sigma^{-1} A_{i_{0}}(y)}-u(y)\right| \\
\leqq & M r \varphi^{*}\left(\delta^{-1} r\right)\left(f_{c \sigma B} \Phi_{p}(|g|) d \mu\right)^{1 / p}+M r^{1-\varepsilon} \delta^{\varepsilon} \\
\leqq & M r \varphi^{*}\left(\delta^{-1} r\right)\left(\frac{\mu(c \sigma B)}{\mu\left(c \sigma B_{0}\right)}\right)^{-1 / p}\left(f_{c \sigma B_{0}} \Phi_{p}(|g|) d \mu\right)^{1 / p}+M r^{1-\varepsilon} \delta^{\varepsilon} \\
\leqq & M r \varphi^{*}\left(\delta^{-1} r\right)\left(\frac{r}{r_{0}}\right)^{-1}\left(f_{c \sigma B_{0}} \Phi_{p}(|g|) d \mu\right)^{1 / p}+M r^{1-\varepsilon} \delta^{\varepsilon} \\
\leqq & M r_{0} \varphi^{*}\left(\delta^{-1} r\right)\left(f_{c \sigma B_{0}} \Phi_{p}(|g|) d \mu\right)^{1 / p}+M r^{1-\varepsilon} \delta^{\varepsilon} .
\end{aligned}
$$

The proof of Theorem 1 is completed by taking $\delta=1$. 
To prove Corollary 1 in case $n \geqq 2$, with the aid of [3. Section 15.26], we first see that if $u \in W^{1, p}\left(\mathbf{R}^{n} ; \mu\right)$ and $B^{\prime}=B\left(x^{\prime}, r^{\prime}\right) \subset A=B\left(x_{0}, r\right)-B\left(x_{0}, r^{\prime}\right)$, then

$$
\begin{aligned}
|u(x)-b| & \leqq \frac{1}{\mathcal{L}^{n}\left(B^{\prime}\right)} \int_{B^{\prime}}|u(x)-u(y)| d y \\
& \leqq M \int_{A}|x-y|^{1-n}|\nabla u(y)| d y
\end{aligned}
$$

for a.e. $x \in A$, where $b=\left(1 / \mathcal{L}^{n}\left(B^{\prime}\right)\right) \int_{B^{\prime}} u(y) d y$. If $B^{\prime}=B\left(x^{\prime}, r^{\prime}\right)$ with $x^{\prime} \in A=$ $B\left(x_{0}, r\right)-B\left(x_{0}, \sqrt{2} r^{\prime}\right)$, then we can also show that

$$
\begin{aligned}
\left|u(x)-b^{\prime}\right| & \leqq \frac{1}{\mathcal{L}^{n}\left(B^{\prime} \cap A\right)} \int_{B^{\prime} \cap A}|u(x)-u(y)| d y \\
& \leqq M \int_{A}|x-y|^{1-n}|\nabla u(y)| d y
\end{aligned}
$$

for a.e. $x \in B\left(x^{\prime}, r^{\prime}\right)-B\left(x_{0}, \sqrt{5} r^{\prime}\right)$, where $b^{\prime}=\left(1 / \mathcal{L}^{n}\left(B^{\prime} \cap A\right)\right) \int_{B^{\prime} \cap A} u(y) d y$. Hence it follows from (3.2) that

$$
\begin{aligned}
\left|u(x)-b^{\prime}\right| & \leqq \frac{1}{\mathcal{L}^{n}\left(B^{\prime} \cap A\right)} \int_{B^{\prime} \cap A}|u(x)-u(y)| d y \\
& \leqq M \int_{A}|x-y|^{1-n}|\nabla u(y)| d y
\end{aligned}
$$

for a.e. $x \in A$. Taking a finite covering of A by balls $B\left(x^{\prime}, r^{\prime}\right)$ with $x^{\prime} \in A=$ $B\left(x_{0}, r\right)-B\left(x_{0}, \sqrt{2} r^{\prime}\right)$, we have

$$
\begin{aligned}
|u(x)-a| & \leqq \frac{1}{\mathcal{L}^{n}(A)} \int_{A}|u(x)-u(y)| d y \\
& \leqq M \int_{A}|x-y|^{1-n}|\nabla u(y)| d y
\end{aligned}
$$

for a.e. $x \in A$, where $a=\left(1 / \mathcal{L}^{n}(A)\right) \int_{A} u(y) d y$. In view of [3, Section 15.23], we establish

$$
\begin{aligned}
f_{A}\left|u-u_{A}\right| d \mu & \leqq 2 f_{A}|u(x)-a| d \mu(x) \\
& \leqq M r\left(f_{A}|\nabla u|^{p} d \mu\right)^{1 / p}
\end{aligned}
$$

which implies the $p$-Poincaré inequality in our sense. Thus the case $n \geqq 2$ follows from Theorem 1.

In the case $n=1$, we apply the usual Poincaré inequality on intervals. We have

$$
\left|u(x)-u_{I_{i_{0}}}\right| \leqq M \sum_{i=-\infty}^{i_{0}} 2^{i} \mu\left(\tilde{I}_{i}\right)^{-1 / p}\left(\int_{\tilde{I}_{i}}|\nabla u|^{p} d \mu\right)^{1 / p}
$$

where $I_{i}$ denotes the interval $\left(x+2^{i-1}, x+2^{i}\right)$ or $\left(x-2^{i}, x-2^{i-1}\right)$. In the same way as in the proof of Theorem 1, we finally obtain

$$
|u(x)-u(y)| \leqq M r_{0} \varphi^{*}\left(\delta^{-1} r\right)\left(f_{B_{0}} \Phi_{p}(|\nabla u|) d \mu\right)^{1 / p}+M r^{1-\varepsilon} \delta^{\varepsilon}
$$


whenever $\delta>0$ and $x, y \in I=\left(x_{0}-r, x_{0}+r\right) \subset\left(x_{0}-r_{0}, x_{0}+r_{0}\right)=B_{0}$, where $0<\varepsilon<1$ and $M$ is a positive constant (which may depend on $\varepsilon$ ). Hence the case $n=1$ is proved similarly.

Proof of Theorem 2. Modify $u$ as in Theorem 1 so that it is locally $\varphi^{*}$-Hölder continuous on $\mathbf{R}^{n}$. Let $x_{0}$ be a point such that

$$
\lim _{r \rightarrow 0} f_{B\left(x_{0}, r\right)} \Phi_{p}\left(\left|\nabla u(x)-\nabla u\left(x_{0}\right)\right|\right) d \mu(x)=0 .
$$

By Lemma 5, almost every $x \in \mathbf{R}^{n}$ has this property. Set $v(x)=u(x)-u\left(x_{0}\right)-$ $\nabla u\left(x_{0}\right) \cdot\left(x-x_{0}\right)$. Then $\nabla v(x)=\nabla u(x)-\nabla u\left(x_{0}\right)$, and (3.5) yields

$$
\lim _{r \rightarrow 0} f_{B\left(x_{0}, r\right)} \Phi_{p}(|\nabla v(x)|) d \mu(x)=0 .
$$

Applying (3.1) in the proof of Theorem 1 with $v$, for $x \in B\left(x_{0}, r\right), 0<\varepsilon<1$ and $\delta>0$, we have

$$
\frac{|v(x)|}{r}=\frac{\left|v(x)-v\left(x_{0}\right)\right|}{r} \leqq M \varphi^{*}\left(\delta^{-1} r\right)\left(f_{c \sigma B} \Phi_{p}(|\nabla v|) d \mu\right)^{1 / p}+M r^{-\varepsilon} \delta^{\varepsilon},
$$

where $c=2^{4}+1$ and $M=M(\varepsilon)$ does not depend on $\delta, r$ and $x$. Here, taking $\delta^{-1} r=N$ with a positive integer $N$, we obtain

$$
\frac{|v(x)|}{r} \leqq M \varphi^{*}(N)\left(f_{c \sigma B} \Phi_{p}(|\nabla v|) d \mu\right)^{1 / p}+M N^{-\varepsilon} .
$$

Hence it follows from (3.6) that

$$
\limsup _{x \rightarrow x_{0}} \frac{|v(x)|}{\left|x-x_{0}\right|} \leqq M N^{-\varepsilon} .
$$

Letting $N \rightarrow \infty$, we see that the left side is equal to zero, which implies that $v$ is totally differentiable at $x_{0}$.

Remark. The proof of Theorem 1 implies that

$$
\left|u(x)-u\left(x_{0}\right)\right| \leqq M\left|x-x_{0}\right|\left(\sup _{0<r<c \sigma r_{0}} f_{B\left(x_{0}, r\right)} \Phi_{p}(|g|) d \mu\right)^{1 / p}+M\left|x-x_{0}\right|
$$

for all $x \in B\left(x_{0}, r_{0}\right)$. This fact completes the proof of Theorem 2 with the aid of Rademacher-Stepanov theorem (cf. [9, Theorem 3, Chap. VIII]).

\section{REFERENCES}

[1] J. Björn, $L^{q}$-Differentials for weighted spaces, Michigan Math.J. 47 (2000), 151-161.

[2] P. Hajłasz and P. Koskela, Sobolev met Poincaré, Mem. Amer. Math. Soc. 145 (2000). MR 2000j:46063

[3] J. Heinonen, T. Kilpeläinen and O. Martio, Nonlinear potential theory of degenerate elliptic equations, Clarendon Press, 1993. MR 94e:31003

[4] Y. Mizuta, Continuity properties of Riesz potentials and boundary limits of Beppo Levi functions, Math. Scand. 63 (1988), 238-260. MR 90m:31010

[5] Y. Mizuta, Continuity properties of potentials and Beppo-Levi-Deny functions, Hiroshima Math. J. 23 (1993), 79-153. MR 94d:31005

[6] Y. Mizuta, Integral representations, differentiability properties and limits at infinity for Beppo Levi functions, Potential Analysis 6 (1997), 237-276. MR 98k:31007

[7] Y. Mizuta and T. Shimomura, Differentiability and Hölder continuity of Riesz potentials of Orlicz functions, Analysis 20 (2000), 201-223. MR 2001h:46052 
[8] T. Shimomura and Y. Mizuta, Taylor expansion of Riesz potentials, Hiroshima Math. J. 25 (1995), 595-621. MR 97d:31009

[9] E. M. Stein, Singular integrals and differentiability properties of functions, Princeton Univ. Press, Princeton, 1970. MR 44:7280

The Division of Mathematical and Information Sciences, Faculty of Integrated Arts and Sciences, Hiroshima University, Higashi-Hiroshima 739-8521, Japan

Department of Mathematics, Graduate School of Education, Hiroshima University, HigASHI-HiRoshima 739-8524, JAPAN 